\title{
ESTUDIO DESCRIPTIVO: BIOSEGURIDAD EN INSTITUCIONES FORMADORAS EN SALUD
}

\section{DESCRIPTIVE STUDY: BIOSECURITY IN HEALTH INSTITUTIONS}

\author{
Calderón Alemán Doris*, ${ }^{1}$ Tello Larriva Mónica, ${ }^{1}$ Montesinos Rivera Mayra, ${ }^{1}$ Andrade Granda Galo. ${ }^{2}$ \\ ${ }^{1}$ Docente Especialista en la Unidad Académica de Salud y Bienestar, Carrera de Odontología- Sede Azogues. Universidad \\ Católica de Cuenca. Ecuador. \\ ${ }^{2}$ Postgrado en Obstetricia y Ginecología. Universidad de Chile - Hospital Luis Tisné Brousse de Santiago de Chile. Chile. \\ *decalderona@ucacue.edu.ec
}

\begin{abstract}
Resumen
RESUMEN: El objetivo de este estudio es analizar los contenidos teóricos y prácticos de bioseguridad declarados en las asignaturas de la Carrera de Odontología de la Universidad Católica de Cuenca sede Azogues-Ecuador, para establecer el grado de instrucción entregado a los estudiantes en proceso de formación en relación a los protocolos de prevención y precaución. MATERIALES Y MÉTODOS: El estudio fue descriptivo, de corte transversal, mediante una revisión, análisis y evaluación de los antecedentes, desarrollo y ejecución del plan de estudios desde la perspectiva de bioseguridad. Está evaluación se realizó del primer al séptimo ciclo de estudio. Se aplicó un instrumento de medición modelo encuesta contrastando los contenidos de bioseguridad presentes en estos documentos y la información entregada por los docentes de la carrera desde el punto de vista teórico, práctico y teoría-práctico. Se empleó una estadística descriptiva que incluía frecuencias relativas en comparación con las variables. RESULTADOS: El $100 \%$ de los docentes de la Carrera de Odontología, considera relevante la enseñanza de bioseguridad en la carrera universitaria, el $82 \%$ impartieron conocimientos de bioseguridad, el $78 \%$ de los docentes no disponía de una referencia bibliográfica específica en bioseguridad y el $22 \%$ contaban con guías de bioseguridad del Ministerio de Salud Pública (MSP) del Ecuador. CONCLUSIONES: El papel de un profesor en la formación de profesionales de la salud debe ser completo e incluir el principio básico y fundamental de la bioseguridad en todas las áreas (básica, preclínica y clínica.) Este enfoque garantizará el cumplimiento de las normas nacionales e internacionales de salud en el ejercicio de su profesión.
\end{abstract}

Palabras clave: Educación continua, exposición a agentes biológicos, organizaciones de normalización profesional.

\begin{abstract}
Summary: The objective of this study is to analyze the theoretical and practical contents of biosecurity declared in the subjects of the Odontology Career of the Catholic University of Cuenca, Azogues-Ecuador, to establish the degree of instruction delivered to students in the process of formation in relation to the prevention and precaution protocols. MATERIALS AND METHODS:The study was descriptive, cross-sectional, through a review, analysis and evaluation of the background, development and execution of the curriculum from a biosecurity perspective. This evaluation was carried out from the first to the seventh study cycle. A survey model measuring instrument was applied, contrasting the biosecurity contents present in these documents and the information given by the professors of the degree from the theoretical, practical and theoreticalpractical points of view. A descriptive statistic was used that includes relative frequencies in comparison with the variables. RESULTS: The 100\% of teachers in the Dentistry Career considers biosafety education relevant in the university career, $82 \%$ taught biosafety knowledge, $78 \%$ of teachers did not have a specific bibliographic reference on biosafety and $22 \%$ had biosafety guidelines from the Ministry of Public Health (MSP) of Ecuador. CONCLUSIONS: The role of a teacher in the training of health professionals must be complete and include the basic and fundamental principle of biosecurity in all areas basic, preclinical and clinical. This approach will ensure compliance with national and international health standards in the exercise of their profession.
\end{abstract}

Key words: Education continuing, exposure to biological agents, professional review organizations.

\section{INTRODUCCIÓN}

El conocimiento de normas de bioseguridad en instituciones de salud, cumple un rol fundamental en la preven- 
ción y reducción del riesgo de transmisión de enfermedades infectocontagiosas en profesionales y pacientes, vulnerables a sufrir accidentes por exposición a fluidos corporales; la bioseguridad comprende la protección de la salud humana y del ambiente con respecto a los riesgos conocidos y/o percibidos en la técnica empleada. El profesional de la salud debe proyectar y garantizar que las normas y protocolos sean aplicados en toda actividad clínica, médica y odontológica en el paciente y el operador. ${ }^{1-3}$ El manual de bioseguridad de la Organización Mundial de la Salud (OMS) promueve su ejecución como parte de programas nacionales que resguarden la salud de las personas en todos los países. ${ }^{4}$ El MSP en el Ecuador, es regulador de los procesos de bioseguridad, dispone del manual de normas para la red de servicios de salud que debe implementarse a nivel nacional, para generar un ambiente seguro en el trabajo y crear una cultura organizacional altamente comprometida con el autocuidado del personal de salud y los usuarios. Diferentes estudios señalan la importancia de poner en práctica las normas de bioseguridad en el área odontológica, por el elevado porcentaje de accidentes ocupacionales que obligan a mantener entornos resguardados y sólidos para el desempeño óptimo y adecuado de las prácticas pre-profesionales durante la formación universitaria. ${ }^{5-8}$ El objetivo fue analizar los contenidos teóricos y prácticos de bioseguridad en las asignaturas de la Carrera de Odontología de la Universidad Católica de Cuenca, sede Azogues-Ecuador, para establecer el grado de instrucción entregado a los estudiantes en proceso de formación.

\section{MATERIALES Y MÉTODOS}

Se realizó un estudio observacional, retrospectivo, de corte transversal donde se analizó el contenido relacionado con temas de bioseguridad en 38 sílabos de las asignaturas impartidas desde primero a séptimo ciclo de la malla curricular actual de la Carrera de Odontología de la Universidad Católica de Cuenca, sede Azogues-Ecuador. Mediante la aplicación de un instrumento de medición en la segunda fase del estudio, se empleó un modelo de encuesta aplicado en un estudio similar ${ }^{9}$ contrastando los contenidos de bioseguridad presentes en estos documentos y la información entregada por los docentes de la carrera. Se utilizó para la base de datos Excel, Microsoft 2000 y una estadística descriptiva que incluyó frecuencias relativas, para conocer el grado de instrucción de los estudiantes en temas de bioseguridad contemplados en programas de clases teóricas, prácticas y teórico-prácticas. Las disciplinas se agruparon en tres partidas, codificadas de la siguiente manera:

- Odontología Formación Básica Fundamentación Teórica (OFBTF).

- Odontología Formación Profesional Avanzada (OFPA).

- Odontología Titulación Epistemología y Métodos de Investigación (OTEMI).

\section{RESULTADOS:}

Se aplica la encuesta al total del universo, 13 docentes titulares a tiempo completo y 38 asignaturas de la Carrera de Odontología de la Universidad Católica de Cuenca sede Azogues-Ecuador. En la tabla 1, se distribuyeron cada una de las disciplinas de primero a séptimo ciclo (octavo a décimo ciclo no han iniciado aún en la carrera), se detalla cuántas corresponden a los diferentes códigos señalados en materiales y métodos.

\begin{tabular}{ll}
\hline $\begin{array}{c}\text { Arcada Superior } \\
\text { Derecha }\end{array}$ & \multicolumn{1}{c}{$\begin{array}{c}\text { Arcada Superior } \\
\text { Izquierda }\end{array}$} \\
\hline $\mathbf{5 1}=$ Corona de & $\mathbf{6 1}=$ Corona de \\
Zirconio $+\mathrm{AO}$ & Zirconio $+\mathrm{AO}$ \\
$\mathbf{5 2}=$ Resina & $\mathbf{6 2}=$ Observación \\
$\mathbf{5 3}=$ Observación + & $\mathbf{6 3}=$ Observación + \\
TS & TS \\
$\mathbf{5 4}=$ Sellador de & $\mathbf{6 4}=$ Resina + TS \\
Fosetas y Fisuras + & \\
TS & \\
$\mathbf{5 5}=$ Resina + TS & $\mathbf{6 5}=$ Resina + TS \\
\hline
\end{tabular}

\begin{tabular}{|c|c|}
\hline $\begin{array}{c}\text { Arcada Inferior } \\
\text { Derecha }\end{array}$ & $\begin{array}{c}\text { Arcada Inferior } \\
\text { Izquierda }\end{array}$ \\
\hline 81 = Observación & 71 = Observación \\
\hline $\begin{array}{l}82=\text { Observación } \\
83=\text { Observación }+\end{array}$ & $\begin{array}{l}72=\text { = Observación } \\
73=\text { Observación }+\end{array}$ \\
\hline & $\begin{array}{l}\text { TS } \\
74=\text { Pulpotomia }+\end{array}$ \\
\hline $\begin{array}{l}\text { Corona de Zirconio } \\
+\mathrm{AO} \\
85=\text { Pulpectomia }\end{array}$ & $\begin{array}{l}\text { Corona de Zirconio } \\
+\mathrm{AO} \\
75 \text { = Pulpotomia }+\end{array}$ \\
\hline $\begin{array}{ll}\text { (Vitapex) }+ \text { Corona } \\
\text { de } & \text { Zirconio }+\end{array}$ & $\begin{array}{l}\text { Corona de Zirconio } \\
+\mathrm{AO}\end{array}$ \\
\hline
\end{tabular}

Tabla 1. Distribución de asignaturas por ciclo y código.

El $100 \%$ de la planta docente considera relevante la enseñanza de bioseguridad en la carrera universitaria, los principios por los cuales se destaca de manera importante este proceso se detalla en la Tabla 2.

Se estima que el $82 \%$ de docentes imparten conocimientos de bioseguridad en la carrera universitaria (Gráfico 1).

Los fundamentos de la entrega de conocimientos de bioseguridad a los estudiantes de la Carrera de Odontología desde el primero al séptimo ciclo se puntualizaron en la Tabla 3. 
PRINCIPIOS DECLARADOS POR LOS DOCENTES UNIVERSITARIOS

Base fundamental para atención a pacientes.
Evitar exposición a riesgos con materiales
infecciosos.
Evitar infecciones.
Evitar infecciones cruzadas.
Evitar accidentes por exposición a material
infeccioso.
Utilizar protocolo para cualquier procedimiento
quirúrgico.
Garantizar condiciones adecuadas que no generen
riesgos para el estudiante y para el paciente.
Indispensable en el proceso de formación de los
estudiantes y parte fundamental de la carrera.
Garantizar la calidad de atención.
Precautelar la seguridad del paciente y operador
según normas internacionales.
Proteger de la integridad y salud de tutores,
pacientes y estudiantes en formación.
Reducir el riesgo de transmisión de
microorganismos.

Tabla 2. Razones por las cuales se considera relevante la enseñanza de bioseguridad en la carrera universitaria.

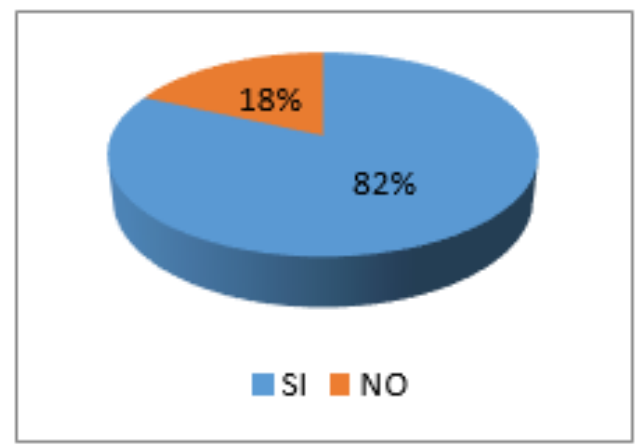

Grf. 1. Proporción de conocimientos de bioseguridad impartidos por los docentes a los estudiantes.

En la Tabla 4 se precisaron los contenidos de bioseguridad que se imparten en las diferentes asignaturas de la Carrera de Odontología, las respuestas se asociaron según la codificación descrita anteriormente en 3 grupos: OFBTF, OFPA, OTEMI

El $70 \%$ de los contenidos de bioseguridad se encontra-

\section{¿POR QUÉ?}

SI NO

Son alumnos en formación y la Debe ser impartida como bioseguridad es un eje cátedra independiente o en fundamental en este proceso. seminarios.

Evitar accidentes $y$ proporcionar ambientes seguros en las áreas de trabajo.

En el caso de materias básicas, son de tipo descriptivo, y no existe manipulación de material infeccioso.

\section{Son protocolos que garantizan}

éxito en los tratamientos.

Contacto directo con fluidos

corporales (saliva).

Prevención de infecciones

cruzadas, sobre todo en el área

quirúrgica.

Es necesario enfatizar en el

control de manipulación,

almacenamiento y desechos de

materiales dentales.

Por ética relacionada

directamente con la

responsabilidad.

Tabla 3. Principios considerados por los docentes, que justifican el por qué imparten conocimientos de bioseguridad en sus asignaturas.

ban declarados en los sílabos correspondientes a las diferentes asignaturas (Gráfico 2).

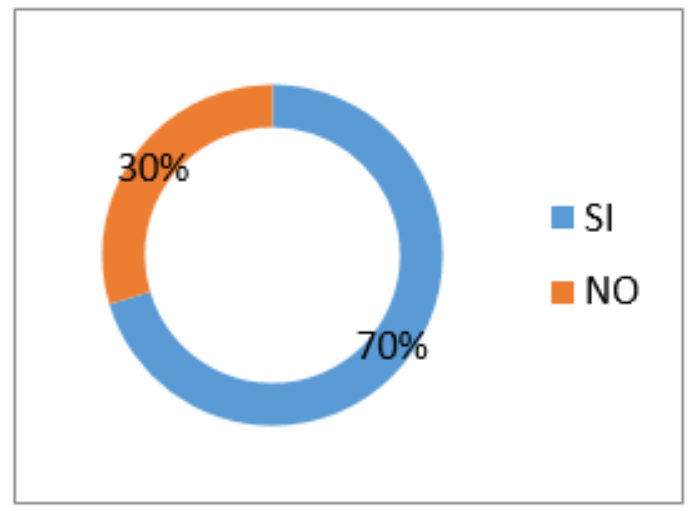

Grf. 2. Contenidos de bioseguridad que se encuentran declarados en el sílabo.

En el Gráfico3, se detalló la distribución de los contenidos de bioseguridad en los diferentes componentes, pudiendo detectar el $42 \%$ en programas prácticos y teórico-prácticos, el $16 \%$ corresponde al programa teórico.

Los contenidos de bioseguridad que no han sido declarados en los sílabos pero que los docentes manifestaron 


\begin{tabular}{|c|c|c|}
\hline OFBTF & OFPA & OTEMI \\
\hline $\begin{array}{l}\text { Bioseguridad en el } \\
\text { laboratorio. }\end{array}$ & $\begin{array}{l}\text { Biaseguridad al } \\
\text { momento del examen } \\
\text { bucal del paciente. }\end{array}$ & Esterilización \\
\hline Espaclos de riesgo. & $\begin{array}{l}\text { Esterilización del } \\
\text { instrumental } \\
\text { odontológico. }\end{array}$ & Asepsia \\
\hline $\begin{array}{l}\text { Guía de trabajo en el } \\
\text { laboratorio de quimica. }\end{array}$ & $\begin{array}{l}\text { Maneja de tejidos, } \\
\text { muestras de biopsia. } \\
\text { Limpieza y desinfección. }\end{array}$ & Antisepsia \\
\hline $\begin{array}{l}\text { Normas de trabajo e } \\
\text { instrumental bisico del } \\
\text { laboratorio } \\
\text { microbiold́gico. }\end{array}$ & $\begin{array}{l}\text { Criterios de desinfección, } \\
\text { descontaminación. }\end{array}$ & \\
\hline $\begin{array}{l}\text { Tratamiento del } \\
\text { instrumental } \\
\text { recuperable, materiales } \\
\text { de un solo uso ut llicados } \\
\text { en la práctica } \\
\text { odontológica. }\end{array}$ & $\begin{array}{l}\text { Uso de uniforme, } \\
\text { instrumentos de } \\
\text { pratección personal, } \\
\text { preparación de mesas de } \\
\text { trabajo e instrumental, Y } \\
\text { preparación de } \\
\text { pacientes. }\end{array}$ & \\
\hline $\begin{array}{l}\text { Agentes fisicos: } \\
\text { esterilización, } \\
\text { desinfección. }\end{array}$ & $\begin{array}{l}\text { Maneja de pacientes con } \\
\text { VIH, manejo } \\
\text { instrumental } \\
\text { periodontal. }\end{array}$ & \\
\hline \multirow[t]{5}{*}{ Agentes quimicos. } & $\begin{array}{l}\text { Maneja de desechos Y } \\
\text { material quirúrgico. }\end{array}$ & \\
\hline & $\begin{array}{l}\text { Asepsia y antisepsia, del } \\
\text { paciente, ciru/,ana, } \\
\text { instrumental, quirófano, } \\
\text { distribudión del personal } \\
\text { en el quirdfana. }\end{array}$ & \\
\hline & $\begin{array}{l}\text { Maniobras previas a la } \\
\text { atencián del paciente, } \\
\text { campa aperatorio. }\end{array}$ & \\
\hline & $\begin{array}{l}\text { Manipulación de } \\
\text { biamateriales. Control de } \\
\text { infecciones, procesos de } \\
\text { desinfección de } \\
\text { materiales dentales } \\
\text { auniliares. }\end{array}$ & \\
\hline & $\begin{array}{l}\text { Contral de infeccián en } \\
\text { raciografla dental. }\end{array}$ & \\
\hline
\end{tabular}

Tabla 4. Contenidos de bioseguridad que se imparten en la Carrera de Odontología de la Universidad Católica de Cuenca, Sede Azogues-Ecuador.

impartir en sus asignaturas, correspondieron a un $63 \%$ del programa teórico-práctico, $25 \%$ práctico y $12 \%$ a teórico (Gráfico 4).

El $26 \%$ de los docentes indicaron que la enseñanza de bioseguridad es sistemática a lo largo del curso y el $74 \%$ la realizaron en un bloque determinado (Gráfico 5).

En el Gráfico 6, el $78 \%$ de los profesionales no disponía de una referencia bibliográfica específica de bioseguridad, utilizaron la bibliografía base para cada asignatura, con insertos de la temática. El $22 \%$ disponía de guías de bioseguridad del MSP del Ecuador.

El $56 \%$ de los docentes no impartieron leyes vigentes sobre bioseguridad, trabajaron con contenidos insertos en la bibliografía de cada asignatura, el $44 \%$ manejaron leyes vigentes relacionadas con el manejo de material infeccioso y desechos cortopunzantes (Gráfico 7).

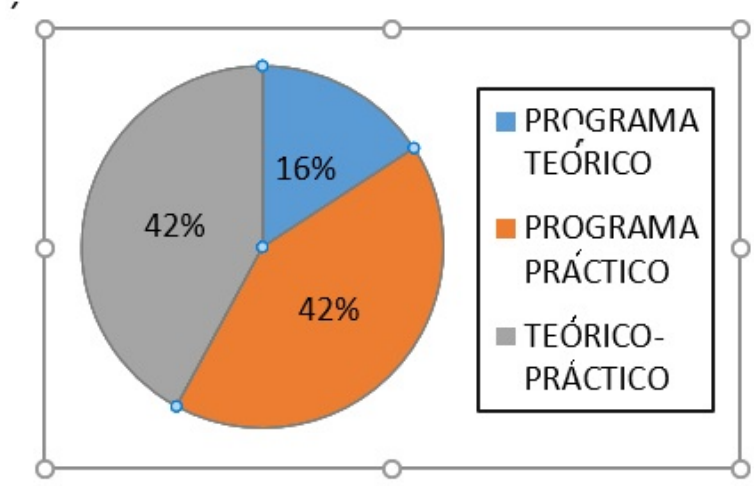

Fuente: Base de recolección de datos Autor: Dra. Doris Calderón Alemán.

Grf. 3. Distribución de los contenidos de bioseguridad en los programas teórico, práctico y teórico-práctico, declarados en el sílabo.

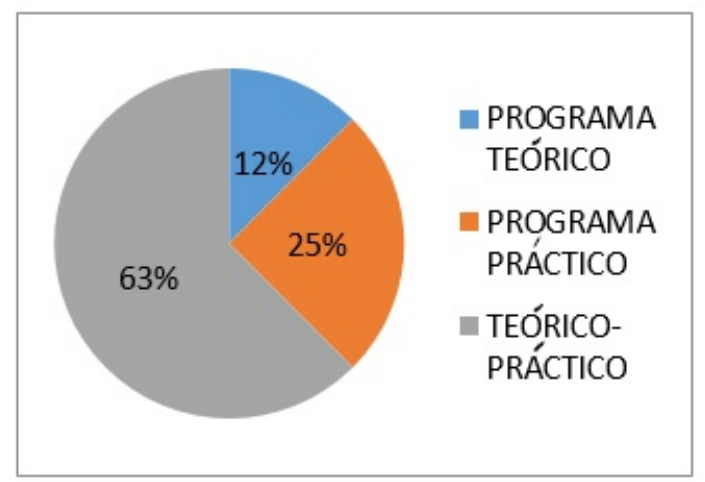

Grf. 4. Distribución de los contenidos de bioseguridad en los programas teórico, práctico y teórico-práctico, que no se encuentran declarados en el sílabo.

\section{DISCUSIÓN}

El docente universitario, juega un rol importante en la formación de los estudiantes para la ejecución de las acciones informativas y formativas que reduzcan y/o eviten posibles riesgos por exposición a fluidos corporales. En estudios similares, se recomienda promover el desarrollo de investigaciones para intervenir en procesos de prevención-enseñanza en los estudiantes debido a que, la aplicación de normas o protocolos de bioseguridad, queda sujeta únicamente a los valores y ética del profesional. Se ha comprobado que en ocasiones es el operador quién no emplea las normas de bioseguridad, siendo el responsable directo de las infecciones cruzadas, enfermedades como el virus de la Hepatitis B y C, virus Herpes Simple, virus de la Inmunodeficiencia Humana (VIH), son patologías transmisibles cuya propagación dependerá de las medidas de bioseguridad que aplique el profesional de la salud. ${ }^{10-12}$ En esta investigación, los docentes 


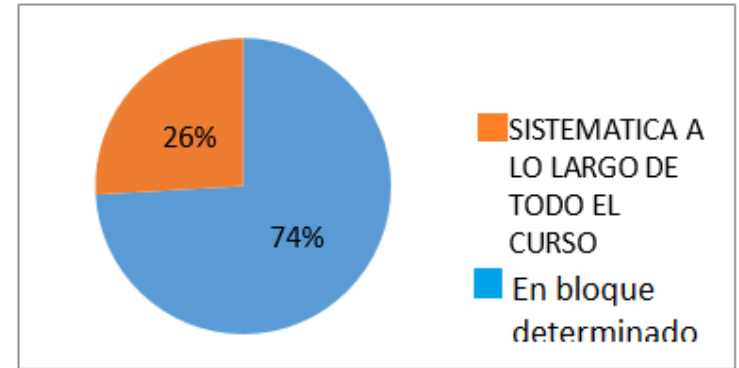

Grf. 5. Cómo es la enseñanza de bioseguridad durante el ciclo académico.

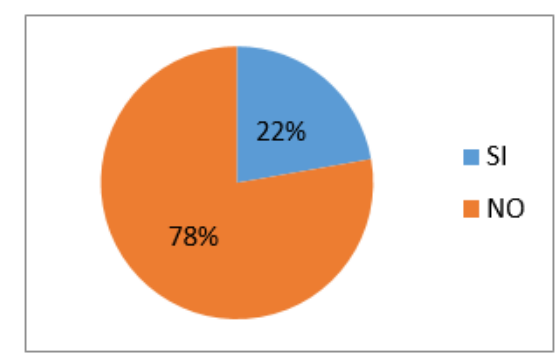

Grf. 6. Disponibilidad de referencias bibliográficas sobre temas de Bioseguridad.

consideraron a la bioseguridad como un tema relevante en el proceso de enseñanza-aprendizaje durante la carrera universitaria al reflejarse que el $82 \%$ de los docentes impartieron conocimientos de bioseguridad, el $70 \%$ de los contenidos se declararon en los sílabos y se abordaron mayoritariamente en programas teórico-prácticos, netamente prácticos y cercanos a la realidad. Estos resultados no se ajustan a situaciones observadas en otras instituciones educativas de nivel superior; en la Universidad Central de Venezuela ${ }^{13}$ y en la Universidad Peruana Cayetano Heredia, ${ }^{14,15}$ se enfatizó que las nociones de bioseguridad durante el proceso de formación universitaria, no son las suficientes, determinando sugerencias que permitan mejorar el acceso a este tema durante el ejercicio profesional.

El estudio realizado analiza el punto de vista de cada docente lo que constituye una línea base, posteriormente se realizará un seguimiento desde el ámbito estudiantil para validar si efectivamente los conocimientos impartidos por los docentes son aplicados por los estudiantes durante su práctica clínica, lo que permitirá establecer si están verdaderamente involucrados en los procesos de bioseguridad y la problemática que conlleva el no cumplimiento de los protocolos, los cuales deberán ser incluidos en los programas curriculares de las entidades universitarias con el objetivo de que los futuros profesionales garanticen su ejecución antes, durante y después de cada intervención odontológica. Como sugerencia se plantea a las autoridades universitarias, incluir

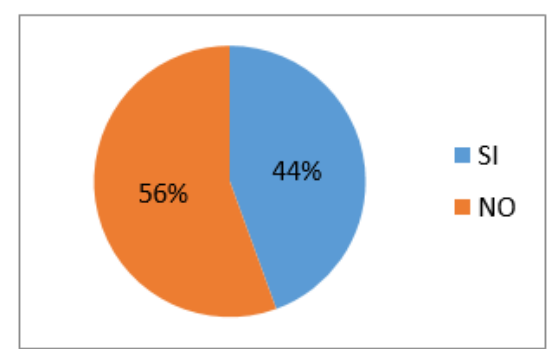

Grf. 7. Proporción de docentes que imparten leyes vigentes en contenidos de bioseguridad.

referencias bibliográficas específicas de bioseguridad en los sílabos de todas las asignaturas, básicas y profesionalizantes, una opción sería el manual de normas de bioseguridad en la red de servicios de salud en el Ecuador, considerado como la base para el cumplimiento en todas las instituciones de salud. El entrenamiento estudiantil en bioseguridad con fines preventivos en todas las asignaturas y clínicas, buscará nuevas estrategias, conocimientos y experiencias que ayuden a definir puntos básicos fundamentales que se practiquen en la rutina diaria, de tal manera que la docencia debe contribuir de manera integral en la formación de los futuros profesionales. $3,11,15$

\section{CONCLUSIÓN}

El rol del docente en la formación de profesionales de la salud es integral e incluye como principio básico y fundamental, la enseñanza de la bioseguridad en todas las áreas: básicas, preclínicas y clínicas; garantizando la aplicación de normas nacionales e internacionales por parte de los estudiantes universitarios, que les permita brindar atención de calidad en el ejercicio de su profesión. Se recomienda capacitar a los estudiantes de odontología sobre las normas y protocolos de bioseguridad antes y durante la realización de sus prácticas pre profesionales así como implementar controles rigurosos para el cumplimiento de las mismas tanto para el estudiante como para el profesional docente.

Conflicto de intereses y financiamiento Los autores declaran no tener conflicto de intereses, haber cumplido con los requisitos de autoría y haber autofinanciado este artículo.

\section{Referencias}

1 Soto V, Olano E. Conocimiento y cumplimiento de medidas de bioseguridad en personal de enfermería. Hospital Nacional Almazor Aguinaga. Anales de la Facultad de Medicina. 2004; 65(2): 103-110. Documento disponible. 
2 Burachik M. Bioseguridad de Organismos Genéticamente modificados - Marcos Regulatorios. Biotecnología. 2004; 387-398.

3 Velazco N, Pino D.Bioseguridad en la Docencia Odontológica. Rev. Odontológica de los Andes. 2014; 9 (1): 51-54.

4 Organización Mundial de la Salud. Manual de Bioseguridad en el Laboratorio. 2005. Disponible en: documento disponible.

5 Manual de Normas de Bioseguridad para la red de servicios de salud en el Ecuador. Ministerio de Salud Pública. Gobierno Nacional de la República del Ecuador. documento disponible.

6 Arrieta K, Díaz S, González F. Prevalencia de accidentes ocupacionales y factores relacionados en estudiantes de odontología. Rev. Salud Pública. 2013; 15 (1): 23-31.

7 Acosta B. Atención al paciente VIH/SIDA: Legislación y bioseguridad odontológica en Colombia. Acta Bioethica. 2006; 12(1), 23-28.

8 Elucir G, Caffer N, Malaguti E,Canini S,MarinR,Hayashida M, et.al.Accidentes con material biológico y la vacunación contra la hepatitis B en estudiantes del área de la salud. Rev. Latino-Americana Enfermagem. 2008; 16 (3):104105.http://dx.doi.org/10.1590/S0104-11692011000200015.

9 Zárate A, Rezzonico M, Castillo M, Castillo G, Castillo B, Bregains L, et.al. Bioseguridad e higiene en la formación del odontólogo. Acta Odontológica Venezolana. 2009; 47 (1): 1-7.

10 Hernández A, Montoya J, Simancas M. Conocimientos, prácticas y actitudes sobre bioseguridad en estudiantes de Odontología. Revista Colombiana de Investigación en Odontología. 2012; 3 (9): 148-157.

11 Irribarra R. La Bioética como soporte de la Bioseguridad. Acta Bioeth. 2006; 12 (1): 29-34.

12 Zenteno P. Bioseguridad en Odontología. Rev. Act. Clin. Med. 2011; 15: 818-821.

13 Polo A, Pinto L, Mago H, Domínguez A. Conocimientos sobre bioseguridad en estudiantes de medicina. II Simposio Latinoamericano y del Caribe. Caracas. Venezuela, 2003.

14 Flores Ch, SamalvidesF. Conocimiento sobre bioseguridad en estudiantes de medicina de una universidad peruana. Rev Med Hered. 2005; 16 (4): 253-259.

15 Licea Y, Rivero M, Solana L, Pérez K.Nivel de conocimientos y actitud ante el cumplimiento de la bioseguridad en estomatólogos. Rev. Ciencias Médicas La Habana. 2012; 18 (1): 80-90.https://doi.org/10.22519/21455333.369.

Recibido: 18 de enero de 2017.

Aceptado: 16 de marzo de 2017. 